$+{ }^{+}+{ }^{+}+$La revue pour l'histoire du CNRS

POUR LHISTOIRE DU CNRS $\quad 24 \mid 2009$

Soixante-dixième anniversaire du CNRS

\title{
La preuve par les urnes
}

Jean Chiche

\section{(2) OpenEdition}

Journals

Édition électronique

URL : https://journals.openedition.org/histoire-cnrs/9070

DOI : 10.4000/histoire-cnrs.9070

ISSN : 1955-2408

Éditeur

CNRS Éditions

Édition imprimée

Date de publication : 5 octobre 2009

ISSN : 1298-9800

Référence électronique

Jean Chiche, "La preuve par les urnes », La revue pour l'histoire du CNRS [En ligne], 24 | 2009, mis en ligne le 05 octobre 2009, consulté le 20 mai 2021. URL : http://journals.openedition.org/histoire-cnrs/ 9070 ; DOl : https://doi.org/10.4000/histoire-cnrs.9070

Ce document a été généré automatiquement le 20 mai 2021.

Comité pour l'histoire du CNRS 


\title{
La preuve par les urnes
}

\author{
Jean Chiche
}

Interview

1 La revue pour l'histoire du CNRS. Tout a commencé par les mathématiques.

2 Jean Chiche. Après les classes préparatoires scientifiques, j'ai suivi un cursus universitaire classique en mathématique à Paris VI et Paris VII. Je me suis spécialisé en statistique mathématique avec un DEA en analyse des données. Déjà attiré par les sciences sociales, j'ai assisté aux cours d'ethnologie de Robert Jaulin. Ma thèse portait sur le comportement non verbal d'autistes en situation d'apprentissage, en étroite collaboration avec des médecins de la faculté de Montpellier. En décryptant les images des séances, j'ai observé les postures, défini les interactions de groupe et fait des propositions d'accompagnement des autistes visées par un psychologue. Puis, j'ai intégré l'Institut supérieur de statistique de l'université Pierre-et-Marie-Curie. La revue... Comment passe-t-on des mathématiques à la vie politique ?

4 J. C. Au Laboratoire d'informatique des sciences de l'homme, on pratiquait déjà le culte de l'interdisciplinarité! On partageait des méthodes, des idées, du savoir-faire informatique... J'ai bénéficié du soutien de Pierre-Olivier Flavigny et de mes amis de l'association pour le développement et la diffusion de l'analyse des données (Addad). Tout en étant à la FNSP, je souhaitais explorer d'autres pans des sciences sociales dans un univers médical. J'ai rejoint François Raveau, neurologue et anthropologue médical qui travaillait avec Jacques Ruffié au Collège de France et avait succédé à Roger Bastide au Centre de recherche sur les dysfonctions de l'adaptation (Centre de l'EHESS). À l'interface entre épidémiologie et anthropologie, j'ai parcouru le monde pour mener des enquêtes sur le terrain: études statistiques sur la maladie mentale vue par les Chinois, la mort subite du nourrisson par les Thaïs ou les Cambodgiens, les représentations du sang dans différentes cultures... La revue... Vous avez ensuite contracté le « virus électoral ».

6 J. C. En parallèle, j'ai travaillé sur les enquêtes électorales, encore rares à l'époque, et sur la mise au point d'une base de données électorale française unifiée à tous les niveaux géographiques, avec un logiciel convivial et facile à utiliser (EDEN). À partir des 
fichiers établis par mes collègues (Élisabeth Dupoirier, Gérard Grunberg, Daniel Boy, Alain Lancelot...), j'ai essayé d'y asseoir une certaine cohérence avec des codes, des dictionnaires, un logiciel adapté aux besoins (analyse, typologie). Et une prédilection pour la géométrie et le côté visuel des choses (nuages de points, sélection de nuages, colorisation). Après dix années passées dans le milieu médical, j'ai été affecté au Cevipof comme agent du CNRS en 1987. Loin d'abandonner les méthodes de la statistique mathématique et de l'informatique, j'ai créé en 2000 le groupe Magda (Méthodes d'analyse géométrique des données appliquées) avec Brigitte Leroux.

8 J. C. Faute de moyens, les enquêtes quantitatives sont sous-traitées à des instituts privés. En 2007, étant donné l'actualité politique chargée (présidentielle et législatives), nous avons obtenu des crédits conséquents pour étudier le comportement électoral des Français. La première enquête initiée en 2006 s'est déroulée en quatre vagues, Le baromètre politique français, avec 5600 personnes interviewées à chaque fois. La deuxième en 2007, a permis de constituer Le panel électoral français pour comprendre la dynamique des choix électoraux en temps de campagne : 4000 personnes ont été interrogées en face à face avant le premier tour; puis par téléphone entre les deux tours, après le second tour et après les législatives.

\section{$9 \quad$ La revue... Les modes de recueil sont-ils satisfaisants?}

10 J. C. Le manque de disponibilité des gens, la difficulté à les rencontrer, la fiabilité des numéros de téléphone fixes... orientent les enquêteurs vers des mixed methods. La méthode la moins onéreuse et la plus prometteuse est évidemment Internet. Je travaille actuellement sur des comparaisons structurelles et inductives pour savoir si les enquêtes en ligne supplanteront (pour des raisons de coût) les méthodes traditionnelles ou les compléteront.

Qui se cache derrière l'écran?

11 Comment s'assurer de l'identité de l'internaute? D'abord, on n'interroge pas les visiteurs occasionnels. Certains instituts gèrent des bases à partir d'informations émanant de sites commerciaux, de catalogues de supermarchés, de la vente en ligne de billets de train... Les Américains, les Canadiens ou les Japonais sont « rémunérés » sous une forme symbolique, les insentive (bons d'achat). Quand on métisse des échantillons contrôlés, vérifiés et produits par des professionnels, les réponses sont honnêtes, fiables et déployées. Internet évite l'effet enquêteur et permet de mieux capter l'attention de l'internaute en lui présentant des photos, des vidéos... L'internaute répond à son rythme en passant plus ou moins de temps et en revenant sur certaines questions. Le résultat n'est biaisé que si les questions posées sont liées à un sujet très conjoncturel et dont les résultats ne sont intéressants que pour la journée. Reste, bien sûr, la question de la représentativité des individus équipés ou ayant accès à Internet. Internet, un allié d'avenir pour les partis politiques Les partis politiques utilisent de plus en plus Internet pour communiquer. Ces pratiques américaines qui interrogent les citoyens sur les enjeux électoraux ont fait leur apparition en France il y a cinq ans. C'est du marketing politique ou une forme de démocratie participative (net democracy) reprise par Ségolène Royal et Nicolas Sarkozy en 2007. Les politiciens américains infléchissent leur discours en fonction des enquêtes. Les images des deux candidats de 2007 ont eu un impact essentiel : la proximité aux gens, la communication, l'inquiétude plus que le positionnement politique. Un débat 
entre deux tours ou à la veille d'une élection ne fait que conforter les choix. Il peut abîmer l'image d'un candidat sans pour autant changer les intentions de vote.

Portrait-robot de l'électeur

Difficile de dessiner un profil type des personnes interviewées. La norme scientifique d'un échantillon repose sur un tirage aléatoire à partir d'une base de sondage (strates, grappes, multilevel). Ensuite, on procède à un calage d'échantillons, pratique courante à l'Insee, l'Ined, ou dans les instituts publics qui disposent de bases de sondages crédibles. Or, une loi de 1945 interdit à l'Insee de fournir des fichiers d'adresses, y compris à la recherche publique. Nous ne disposons donc pas de base de sondage propre. D'où la rareté des sondages aléatoires à cause du coût prohibitif. L'European Social Survey (ESS), protocole de sciences sociales et de sciences humaines le plus intégré au niveau européen, unifie les questionnaires, les modes de recueil, élimine au mieux les biais, met en place des contraintes méthodologiques très fortes des résultats comparables et analysables. Mais les coûts restent prohibitifs! On avoisine les 450000 euros, alors que 1500 interviews par téléphone coûtent environ 35000 euros, les face à face 90000 euros, et Internet, moins de 10000 euros.

Paris, une exception?

Jusqu'en 2001, le vote des Parisiens et des Franciliens a beaucoup surpris. Nous travaillons sur la base des bulletins réellement dépouillés dans les urnes. Et l'exercice est périlleux avec une clôture des bureaux de vote à 20 heures dans les grandes villes ! À la fin du chiraquisme, fin des années 1990, Paris était à contre-courant. On a parlé de gentryfication, de boboïsation... Mais le reste des Français qui habitent dans les grandes agglomérations, de plus de 100000 habitants, se sont mis au diapason. Et en 2008, Paris était le leader d'une certaine mouvance. À Toulouse les pronostics ont été soumis à rude épreuve (Ndlr : Pierre Cohen, candidat PS, a fait basculer la mairie à gauche). Le XIIe arrondissement de Paris a connu la plus forte progression de la gauche.

Des électeurs sous influence?

D'une manière générale, les électeurs ne sont pas influencés par les résultats des sondages. Ils les utilisent comme ils utiliseraient un éditorial, le JT de 20 heures, un article de la presse quotidienne ou hebdomadaire, de la presse quotidienne régionale $(\mathrm{PQR})$. Cet outil d'information et de communication parmi d'autres peut conforter leur décision mais ne pèsera pas plus dans la balance. En 2002, à la veille du premier tour, les sondages prédisaient $20 \%$ d'indécis. Les éditorialistes ont adopté une attitude politiquement correcte en affirmant, «le deuxième tour va opposer Chirac à Jospin. » Dans les derniers sondages, il y a eu un resserrement inférieur à l'intervalle de confiance. Les journalistes auraient dû alerter l'opinion, comme l'a fait Le Monde, en disant: «il est possible que le premier tour crée la surprise dimanche.» C'était statistiquement envisageable. C'est la raison pour laquelle en 2007, les journalistes ont été beaucoup plus prudents.

Machine à voter, 1928. (C) CNRS Photothèque - Fonds ancien

17 Analyse acoustique de trois discours de François Mitterrand. Un savant dosage entre parole et silence. (C) CNRS Photothèque/Danielle Duez 


\section{RÉSUMÉS}

À chaque campagne électorale, les commentateurs politiques en vont de leur analyse des tendances, des intentions de vote, des retournements de situation. Ils utilisent certains outils que les mathématiciens ont inventés à d'autres fins. Jean Chiche fait partie de cette catégorie de chercheurs que l'on trouve là où on ne les attendait pas forcément. Il s'en explique.

\section{AUTEUR}

\section{JEAN CHICHE}

Jean Chiche, docteur en statistique mathématique, est ingénieur de recherche au CNRS au Centre de recherches politiques de Sciences Po Paris (Cevipof). 